\section{Herpes simplex encephalitis}

\section{P G E Kennedy, A Chaudhuri}

\section{Herpes simplex virus encephalitis still has an unacceptably high mortality}

$\mathrm{H}$ erpes simplex virus (HSV) is a human herpesvirus that causes HSV encephalitis (HSE), which is the commonest fatal sporadic encephalitis in humans. ${ }^{12}$ About $90 \%$ of all HSE cases in adults and children are due to HSV-1, while HSV-2 is associated with HSE in neonates, in which there is a disseminated infection, and in immunocompromised patients, such as those with renal transplants or HIV infection. ${ }^{34}$ While the exact incidence of HSE is not known, it has been estimated at about one case per million per year. ${ }^{4}$ This figure is probably an underestimate since about 2000 cases occur annually in the United States. ${ }^{1}$ The neuropathological picture of HSE is characteristic, consisting of an acute necrotising encephalitis that almost always localises, often asymmetrically, to the orbitofrontal and temporal lobes with involvement of the cingulate and insular cortex; neonatal HSE tends to produce a more diffuse pathology. ${ }^{1}$ Untreated, HSE has an extremely high mortality rate at about $70 \%$ with fewer than $3 \%$ of survivors returning to normal function. ${ }^{26}$ Among common central nervous system (CNS) viral infections, mortality in HSE is disproportionately high, taking into account a recent study that showed that HSV infections are responsible for only $11 \%$ of cases compared with $29 \%$ for varicella-zoster virus, ${ }^{7}$ another human herpesvirus that is not associated with such a high mortality.

The neuropathogenesis of HSE has intrigued clinicians and scientists for many years, with two of the key questions relating to, firstly, the very low incidence of the condition in the presence of the widespread carriage of latent HSV in ganglionic tissues in healthy people and, secondly, the propensity of the disease process to localise to the frontotemporal region. About $90 \%$ of normal people are seropositive for HSV-1 indicating past exposure to the virus, a finding that is consistent with the presence of latent HSV- I genomes in the trigeminal ganglia of $85-90 \%$ of people at unselected necropsy. ${ }^{8}$ Latent HSV-l periodically is reactivated, either spontaneously or following various triggering events such as trauma, sunlight, immunosuppression, and $x$-ray irradiation, to cause blisters in the mouth and lips, also known as cold sores. ${ }^{14}$ The question therefore arises as to whether HSE results from reactivation of latent HSV-1 from the ganglia with subsequent spread to the CNS. However, only about $25 \%$ of patients with HSE give a prior history of cold sores, an incidence that is no different from that seen in the normal population. ${ }^{1}$ This issue was clarified by Whitley et al, ${ }^{5}$ who used restriction enzyme analysis of HSV-1 isolates from oral, labial, and brain sites in patients with HSE to show that the latter can result from reactivation of latent HSV but may also follow a primary herpetic infection or a reinfection by a second herpesvirus. ${ }^{5}$ There is also evidence for the presence of HSV genomes in the CNS of normal patients, which may in principle act as another source of reactivated virus. ${ }^{9}$

Regarding the site specificity of HSE, the pathway of viral spread is probably more important than cell-type viral susceptibility. The unique anatomical localisation has been thought to result from entry of the virus via the olfactory pathway with spread along the base of the brain to the temporal lobes, ${ }^{1}$ a view that is supported by the immunocytochemical evidence of HSV antigens in the olfactory tract and cortex, as well as temporal lobes, hippocampus, amygdaloid nucleus, insula, and cingulate gyrus in patients dying from HSE. ${ }^{10}$ Another suggestion is that HSE may result from viral spread from the trigeminal ganglia to the temporal and frontal cortex, ${ }^{11}$ a view that is consistent with this known site of HSV latency.

The index of suspicion of HSE should always be high for a patient presenting with the typical features of encephalitis such as fever, headache, confusion, and clouding of consciousness. More specifically, HSE is typically associated with a constellation of frontotemporal features with aphasia or mutism, personality change, and focal or generalised seizures, and in some cases coma. ${ }^{12}{ }^{13}$ Meningism, focal motor weakness, and occasionally brainstem encephalitis, which may be recurrent, have also been described ${ }^{5} 1^{14}$ The diagnosis of HSE is usually established from the combination of the clinical and investigative features. Magnetic resonance imaging (MRI) provides the most sensitive method of detecting early lesions and is the imaging of choice in $\mathrm{HSE}^{15}$; if MRI is available it should be the first diagnostic step after clinical assessment. Cranial MRI may show evidence of focal oedema in the medial region of the temporal and orbital surface of the frontal lobes, insular cortex, and angular gyrus. ${ }^{15}$ Meningeal and gyral enhancement after gadolinium diethylenetriaminepentaacetic acid (DTPA) administration has also been reported..$^{16}$ However, the MRI may occasionally be normal in HSE. ${ }^{15}$ Computed tomography may be normal for the first few days after the onset of symptoms. ${ }^{8}$ When computed tomography is abnormal it usually shows reduced attenuation in one or both frontal or temporal lobes and sometimes areas of hyperintensity representing small haemorrhages ${ }^{15}$ and with midline shift in about $50 \%$ of cases in one retrospective study. ${ }^{12}$ Where available, single photon emission computed tomography, while not diagnostic, may provide functional imaging evidence of HSE. ${ }^{17}$ The EEG is invariably abnormal in HSE. ${ }^{12}$ While nonspecific slowing may be the only feature early in the illness, the later stages are more likely to be associated with high voltage periodic lateralising epileptiform discharges. If present they are diagnostically useful but they are not specific for HSE. ${ }^{15}$

Examination of the cerebrospinal fluid (CSF) is of considerable diagnostic value in HSE and should always be performed after computed tomography or MRI. The exception, in our view, is where cranial imaging shows evidence of severe cerebral oedema and brain shift, in which case we prefer to delay lumbar puncture until the oedema is reduced with steroids or mannitol because of the risk of brain herniation. While about $5 \%$ of patients have normal CSF, ${ }^{15}{ }^{18}$ the characteristic profile consists of a normal or raised pressure, a lymphocytic pleiocytosis $^{815}$ (typically 10-200 cells/ $\mathrm{mm}^{3}$ ), normal glucose, and increased protein $(0.6$ to $6 \mathrm{~g} / \mathrm{l})$. Red blood cells and xanthochromia may be present in the CSF in some patients but are of no diagnostic value in distinguishing HSE from other causes of encephalitis. ${ }^{8}$ CSF polymerase chain reaction (PCR) for HSV DNA has been a major diagnostic advance and has helped to identify different patterns of HSV infection of the CNS. In an experienced laboratory a PCR test for HSV in the CSF of a patient with HSE during the first week can detect viral DNA in about $95 \%$ of cases. ${ }^{15}{ }^{19}$ False negative results are most likely to occur very early, for example, within the first 24-48 hours, or late, for example, after 10-14 days, during the course of the illness. ${ }^{8}$ The specificity of the PCR test is also excellent, probably over $95 \%$ in experienced laboratories with the ability to avoid contamination, ${ }^{19}$ so that a positive result in the CSF is powerful evidence of HSE. Another great advantage of PCR for HSV detection is that the 
assay can be completed quickly, typically within 24 hours, as opposed to anti-HSV antibody measurements, the results of which become available long after the point at which treatment decisions have to be made. Despite their eventual diagnostic usefulness, antiviral antibody titres may also be difficult to interpret for a number of reasons. ${ }^{20}$ Recently, it has been possible to perform quantitative PCR assays for HSV ${ }^{21}$ and this should be of use both diagnostically and in neuropathogenetic studies.

Brain biopsy in HSE now has only a limited role. Before the advent of acyclovir (see below) brain biopsy was regularly used to make a definitive diagnosis of HSE, as both its sensitivity and specificity are extremely high $(95 \%$ and $>99 \%$, respectively).22 However, the advent of HSV PCR and acyclovir has resulted in few neurologists advocating a brain biopsy to diagnose HSE. However, we consider doing a brain biopsy in a patient with suspected HSE in whom there remains serious doubt about the diagnosis, especially where there is a realistic chance of obtaining an alternative diagnosis by obtaining a specimen of brain tissue. As a result of careful PCR based studies on the CSF of large groups of patients, it is now recognised that about a fifth of patients with HSE have mild or atypical disease, ${ }^{23}$ something that some neurologists had suspected for some time. These patients may have had either HSV-1 or HSV-2 encephalitis, were frequently immunosuppressed, and had an asymmetric HSV infection affecting mainly the non-dominant temporal lobe..$^{23}$

Both the mortality and morbidity of patients with HSE have been dramatically improved by the advent of the nucleoside analogue acyclovir (acycloguanosine) especially if it is initiated early, as soon as the diagnosis of HSE is suspected. ${ }^{64}$ Acyclovir selectively inhibits the HSV specific DNA polymerase and is activated specifically in HSV infected cells. Its non-toxicity to normal cells, which lack the HSV thymidine kinase enzyme, is the basis for its remarkable lack of toxicity in patients. ${ }^{14}$ The standard dose of acyclovir is $10 \mathrm{mg} / \mathrm{kg}$ three times daily for 14 days. While acyclovir is often given for a total of 10 days, we advocate 14 days in PCR proven HSE to decrease the possibility of a relapse, which has been reported after weeks to three months after only 10 days' treatment. ${ }^{15}$ While careful thought should always be given before starting acyclovir in suspected HSE, it is inevitable that this drug will be given to many patients who do not have HSE. Once acyclovir has been started in the latter cases, however, it is reasonable to continue treatment for at least 10 days and to stop treatment only if an alternative diagnosis has been established. Otherwise, a clinical deterioration following cessation of acyclovir may be difficult to interpret. In proven HSE in the immunosuppressed patient the duration of treatment with acyclovir can be extended to 21 days to prevent a relapse. ${ }^{15}$ The cause of relapses of HSE after acyclovir is unclear and a recent study of five cases of apparent relapse of HSE found that the relapses may not have been caused by active viral replication. ${ }^{25}$ In the immunosuppressed patient it is particularly important to exclude cytomegalovirus as a potential factor in causing the encephalitis, since this would make treatment with ganciclovir appropriate ${ }^{26}$ Acyclovir resistance in proven HSE has not been shown to occur in immunocompetent patients, ${ }^{27}$ although HSV resistance to acyclovir has been reported in the immunocompromised. ${ }^{28}$ Whether intravenous acyclovir treatment should be followed by the use of valacyclovir orally ${ }^{26}$ for an extended period is an issue that has yet to be resolved. Despite these remarkable advances in treatment, HSE still has an unacceptably high mortality (20-30\% with acyclovir $)^{624}$ and morbidity. A better outcome is seen in younger patients below the age of 30 years and in those in whom the duration of encephalitis was four days or less and the Glasgow coma score was above 6 when acyclovir was started. ${ }^{13}$ However, patients who have been treated very early in the illness and who appear to have made a good recovery may still be left with significant neuropsychological and neurobehavioural impairment that may leave them permanently disabled and unable to return to work. There is still a pressing need to develop yet more effective treatments for HSE.

J Neurol Neurosurg Psychiatry 2002;73:237238

\section{Authors' affiliations}

P G E Kennedy, A Chaudhuri, Glasgow

University Department of Neurology, Institute of Neurological Sciences, Southern General Hospital, Glasgow G51 4TF, UK

Competing interests: none declared

Correspondence to: Professor P G E Kennedy; P.G.Kennedy@clinmed.gla.ac.uk.

\section{REFERENCES}

1 Johnston RT. Viral infectious of the nervous system, 2nd edn. Philadelphia: Lippincott-Raven, 1998.

2 Whitley RJ. Viral encephalitis. N Engl J Med 1990;323:242-50.

3 DeBasi RL, Tyler KL. Recurrent aseptic meningitis. In: Davis LE, Kennedy PGE, eds. Infectious diseases of the nervous system. Oxford: Butterworth-Heinemann, 2000:445-80.

4 Kennedy PGE. Herpes simplex virus and the nervous system. Postgrad Med 1984;60:253-9.

5 Whitley R, Lakeman AD, Nahmias A, et al. DNA restriction enzyme analysis of herpes simplex virus isolates obtained from patients with encephalitis. N Engl J Med 1982;307:1060-2.

6 Whitley RJ, Alford CA, Hirsch MS, et al. Vidarabine versus acyclovir therapy in herpes simplex encephalitis. N Engl J Med

1986:314:144-9.

7 Koskiniemi M, Rantalaiho T, Piiparinen $\mathrm{H}$, ef al. Infections of the central nervous system of suspected viral origin: a collaborative study from Finland. J Neurovirol 2001;7:400-8.

8 Baringer JR. Herpes simplex virus encephalitis. In: Davis LE, Kennedy PGE, eds. Infectious diseases of the nervous system. Oxford: Butterworth-Heinemann, 2000: 139-64.

9 Baringer JR, Pisani P. Herpes simplex virus genomes in human nervous system tissue analysed by polymerase chain reaction. Ann Neurol 1994;36:823-9.

10 Esiri MM. Herpes simplex encephalitis: an immunohistological study of the distribution of viral antigens within the brain. J Neurol Sci 1982;54:209-26.

11 Davis LE, Johnson RT. An explanation for the localization of herpes simplex encephalitis? Ann Neurol 1979;5:2-5.

12 Kennedy PGE. A retrospective analysis of forty-six cases of herpes simplex encephalitis seen in Glasgow between 1962 and 1985 Q J Med 1988;68:533-40.

13 Whitley RJ, Gnann JW. Viral encephalitis: familiar infections and emerging pathogens. Lancet 2002;359:507-14.

14 Tyler KL, Tedder DG, Yamamoto L, et al. Recurrent brainstem encephalitis associated with herpes simplex virus type 1 DNA in cerebrospinal fluid. Neurology 1995;45:2246-50.

15 Davis LE. Diagnosis and treatment of acute encephalitis. Neurologist 2000:6: 145-59.

16 Demaerel PH, Wilms G, Robberecht W, et al. MRI of herpes simplex encephalitis. Neuroradiology 1992;34:490-3.

17 Launes J, Nikkinen P, Lindroth L, et al. Diagnosis of acute herpes simplex encephalitis by brain perfusion single photon emission computed tomography. Lancet 1988;i:1188-91.

18 Whitley RJ, Soong SJ, Linneman C Jr, et al. Herpes simplex encephalitis: diagnosis, presentation, and outcome. JAMA presentation, and
1989;262:234-9.

19 Lakeman FD, Whitley RJ and the National Institute of Allergy and Infectious Diseases Collaborative Antiviral Study Group. Diagnosis of herpes simplex encephalitis: application of polymerase chain reaction to cerebrospinal fluid from brain-biopsied patients and correlation with disease. J Infect Dis 1995;171:857-63

20 Kennedy PGE. The widening spectrum of infectious neurological disease. J Neurol Neurosurg Psychiatry 1990;53:629-32.

21 Revello MG, Baldanti F, Sarasini A, et al. Quantitation of herpes simplex virus DNA in cerebrospinal fluid of patients with herpes simplex encephalitis by the polymerase chain reaction. Clin Diagn Virol 1997; 7: 183-91.

22 Whitley RJ, Soong SJ, Dolin R, et al. Adenine arabinoside therapy of biopsy-proven herpes simplex encephalitis: National Institute of Allergy and Infectious Diseases Collaborative Antiviral Study. N Engl J Med 1977;297:289-94.

23 Fodor PA, Levin M, Weinberg A, et al. Atypical herpes simplex virus encephalitis diagnosed by PCR amplification of viral DNA from CSF. Neurology 1998;51:554-9.

24 Skoldenberg B, Forsgren $M$, Alestig $K$, et al. Acyclovir versus vidarabine in herpes simplex encephalitis: randomized multicentre study in consecutive Swedish patients. Lancet 1984; ii:707-12.

25 Dennett C, Klapper PE, Cleator GM Polymerase chain reaction in the investigation of 'relapse' following herpes simplex encephalitis. J Med Virol 1996;48: 129-32.

26 Balfour HH Jr. Antiviral drugs. N Engl J Med 1999:340: 1255-68.

27 Griffiths PD. Herpesviruses: from the cradle to the grave. Microbiol Today 2001;28:182-4.

28 Christophers J, Clayton J, Craske J, et al. Survey of resistance of herpes simplex virus to acyclovir in northwest England. Antimicrob Agents Chemother 1998;42:868-72. 